\title{
Manifestações da Síndrome de Burnout e fatores associados entre discentes do curso em odontologia
}

\author{
Manifestations of Burnout Syndrome and associated factors among dentistry students \\ Manifestaciones del Síndrome de Burnout y factores asociados entre los estudiantes de \\ odontología
}

Talita Polyana de Freitas Veloso ${ }^{1 *}$, Crislainy Cardoso Pereira de Cassio², Carlos Alberto Quintão Rodrigues $^{1,2}$, Giulia Emannuele Albuquerque Costa ${ }^{2}$, Luiz Pedro Carvalho Rassilan.

\section{RESUMO}

Objetivo: O estudo teve como objetivo verificar e caracterizar os fatores estressores associados ao Burnout entre discentes do curso de Odontologia. Métodos: Trata-se de um estudo corte transversal, descritivo e com análise quantitativa, foram conduzidas perguntas relacionadas ao estresse no ambiente acadêmico. A variável dependente foi estabelecida a partir dos escores médios do questionário "Estresse no Ambiente Odontológico" sendo classificada em "Abaixo ou igual à média" e "Acima da média" nas análises conduzidas no programa estatístico SPSS ${ }^{\circledR} 20.0$, níveis de significância de $p<0,20$ na análise bivariada e $p<0,05$ na múltipla. $A$ regressão de logística foi conduzida para identificar as associações entre o estresse e os fatores de interesse, estimando-se o intervalo de $95 \%$ de confiança. Resultados: Dentre os 344 participantes, $62 \%$ apresentaram algum grau de estresse e $38 \%$ não estressados. $\mathrm{Na}$ análise bivariada, as condições sociodemográficas e a situação acadêmica atual permaneceram associadas ao estresse, relacionado à etapa clínica do curso, à mudança de cidade, reprovações e quantidade de horas dedicada aos estudos. Conclusão: Verificou-se a necessidade de medidas assistenciais para os acadêmicos que estão com algum grau de estresse para prevenir o aparecimento de doenças.

Palavras Chave: Burnout, Estresse, Estudante, Odontologia.

\section{ABSTRACT}

Objective: The study aimed to verify and characterize the stressors associated with Burnout among students of the Dentistry course. Methods: This is a cross-sectional, descriptive study with quantitative analysis, questions were asked related to stress in the academic environment. The dependent variable was established from the average scores of the questionnaire "Stress in the Dental Environment" being classified as "Below or equal to the average" and "Above the average" in the analyzes conducted in the statistical program SPSS® 20.0 , significance levels of $p<0.20$ in the bivariate analysis and $p<0.05$ in the multiple. Logistic regression was conducted to identify the associations between stress and the factors of interest, estimating the $95 \%$ confidence interval. Results: Among the 344 participants, $62 \%$ had some degree of stress and $38 \%$ were not stressed. In the bivariate analysis, the sociodemographic conditions and the current academic situation remained associated with stress, related to the clinical stage of the course, the change of city, failures and the amount of hours dedicated to studies. Conclusion: There was a need for assistance measures for students who are under some degree of stress to prevent the onset of diseases.

Key words: Burnout, Stress, Student, Dentistry.

\section{RESUMEN}

Objetivo: El estudio tuvo como objetivo verificar y caracterizar los factores estresantes asociados con Burnout entre los estudiantes del curso de Odontología. Métodos: Este es un estudio descriptivo de corte transversal con análisis cuantitativo, se hicieron preguntas relacionadas con el estrés en el entorno académico. La variable dependiente se estableció a partir de los puntajes promedio del cuestionario "Estrés en el entorno dental" que se clasificó como "Por debajo o igual al promedio" y "Por encima del promedio" en los análisis realizados en el programa estadístico SPSS $\AA 20.0$, niveles de significación de $p<0.20$ en el análisis bivariado

\footnotetext{
${ }^{1}$ Universidade Estadual de Montes Claros (UNIMONTES), Montes Claros - MG.

*E-mail: talitapfv@yahoo.com.br

${ }^{2}$ Faculdades Unidas do Norte de Minas (FUNORTE), Montes Claros - MG.
}

SUBMETIDO EM: 4/2020

ACEITO EM: 5/2020

PUBLICADO EM: 7/2020 
y $\mathrm{p}<0.05$ en el múltiplo. Se realizó una regresión logística para identificar las asociaciones entre el estrés y los factores de interés, estimando el intervalo de confianza del 95\%. Resultados: entre los 344 participantes, el $62 \%$ tenía algún grado de estrés y el $38 \%$ no estaba estresado. En el análisis bivariado, las condiciones sociodemográficas y la situación académica actual permanecieron asociadas al estrés, relacionadas con la etapa clínica del curso, el cambio de ciudad, los fracasos y la cantidad de horas dedicadas a los estudios. Conclusión: Se necesitaban medidas de asistencia para los estudiantes que están bajo cierto grado de estrés para prevenir la aparición de enfermedades.

Palabras clave: Burnout, Estrés, Estudiante, Odontología.

\section{INTRODUÇÃO}

Os graduandos em Odontologia vivem inseridos em um ambiente estudantil e laboral permeado por conflitos, exigências, angústias e, muitas vezes, insegurança sobre o futuro. Diante disso, condições adversas como a Síndrome de Burnout (SB) estão cada vez mais prevalentes nesse grupo (NEVES CP, 2014). Burnout traduzido do inglês "burn" quer dizer "queima" e "out" significa "exterior", caracterizando um agrupamento de alterações em estudantes e profissionais que lidam no seu dia-a-dia com outros indivíduos. Essas alterações são facilmente percebidas por outros profissionais da área da saúde (TAVARES KFA, et al., 2014; GOMES RC, 2014).

A SB pode ser definida como uma condição de desgaste ocupacional de caráter psicossocial (SCHMIDT DRC, et al., 2013). Gradativamente, o indivíduo acometido encontra dificuldades de lidar com os fatores estressores interpessoais contínuos e se tornam cada vez mais esgotados emocionalmente, despersonalizados e irrealizados profissionalmente, o que afeta sua produtividade (MASLACH C e JACKSON SE, 1981; PEREIRA AMTB, 2010; DIEHL, L. e CARLOTTO MS, 2015; KOGA GKC, et al., 2015).

As manifestações sintomatológicas da SB entre discentes se constituem em três dimensões: exaustão emocional/cansaço físico, caracterizando-se pela falta de energia, disposição, perda de entusiasmo e criatividade para desenvolvimento de suas tarefas habituais, despersonalização/desumanização, percebida como um sentimento incapacitante em que os graduandos não conseguem interagir com a vida alheia, criando uma "barreira" para que os problemas dos outros indivíduos não sejam capazes de refletir sobre sua própria vida, e eficácia profissional, assinalada como as consequências do cansaço físico e emocional e a desumanização citados anteriormente no qual sobre estes gera se uma percepção de estarem sendo ineficazes como estudantes (PEREIRA AMTB, 2010; VIANA GM, et al., 2014).

Os sintomas físicos associados ao Burnout são: cefaleia, suor excessivo, desânimo, pressão arterial elevada, crises asmáticas, tensão muscular, alterações no sistema gastrointestinal, e hipermenorréia ou oligomenorreia, considerados também como sintomas físicos para as mulheres (VARELLA D, 2016).

Ressalta-se que o fato dos discentes desenvolverem a síndrome pode ser explicado através da mudança que estes enfrentam do ensino médio para o vestibular, e logo para a universidade, a chamada transição da fase adolescente para o adulto, período pelo qual, estes indivíduos estão em formação da sua personalidade e se confrontando com o sentimento de perda das características da infância.

Durante essa passagem, os indivíduos enfrentam outra realidade: quando entram em contato com o ambiente acadêmico, percebem que suas responsabilidades aumentam, e essa transição afeta seu psicológico resultando em respostas emocionais negativas como conflitos interiores, dependência de medicamentos, antissocialíssimo, podendo levar ao etilismo e até mesmo ao suicídio. Outro fator que pode estar associado para o desenvolvimento da SB é os indivíduos submeter a condições de estresse de forma permanente (BORINE RCC, et al., 2015).

A prevalência do estresse psicológico tem sido associada a SB observada por pesquisadores e está vêm sendo considerada como um problema de saúde pública devido às consequências que a mesma traz para a saúde geral dos indivíduos acometidos (CAMPOS JADB, et al., 2012).

Conhecer as condições que expõem os estudantes ao estresse torna-se importante para evitar complicações futuras além do aparecimento da SB. Percebe-se diante das buscas literárias em artigos e 
periódicos sobre o assunto, que o tema é atual e necessita de estudos na área odontológica com abrangência na graduação, pois durante esse período ocorrem diversas mudanças emocionais.

Assim, sabendo da importância de investigar tal condição com o intuito de prevenir a mesma, o objetivo desse estudo foi verificar e caracterizar os fatores estressores associados ao Burnout entre discentes do curso de graduação em Odontologia.

\section{MÉTODOS}

Trata-se de um estudo epidemiológico corte transversal, com caráter descritivo e análise quantitativa, conduzido no período de fevereiro a maio de 2017 entre os graduandos do curso superior em odontologia em uma instituição particular de Montes Claros/MG.

Verificou-se junto à direção da instituição que o número de estudantes devidamente matriculados no curso de Odontologia correspondia a 681 discentes. Com isso, por meio de um cálculo online, foi avaliada uma amostra probabilística para população finita, com estimativa de proporções da ocorrência dos eventos em $50 \%$ da população, grau de confiança de $95 \%$, erro de $5 \%$ e acrescidos $10 \%$ para atenuar possíveis perdas, totalizando uma amostra de 345 acadêmicos devidamente matriculados do primeiro ao décimo período do curso.

A coleta de dados foi realizada a partir de questionários semiestruturados, com 11 questões relacionadas às condições socioeconômicas com as seguintes variáveis: sexo, semestre letivo, idade em anos, estado civil, condição atual de moradia, mudança de cidade, estado ou país para realização do curso, permanência na mesma turma que ingressou no vestibular, reprovação nas disciplinas, fonte de renda, situação de trabalho, horas de estudo dedicadas semanalmente; e as 35 perguntas relacionadas aos fatores estressores associados ao Burnout.

Sendo assim, por meio de pesquisadoras calibradas, os participantes foram convidados a participar do estudo e a responderem ao questionário na plataforma online de Formulários Google, após a apresentação de informações sobre a pesquisa e entrega dos termos de consentimento livre e esclarecido, realizadas na própria Instituição de Ensino, considerando-se o período no qual os educandos não estivessem realizando atividades avaliativas.

Para avaliar os fatores associados a SB, utilizou-se o questionário Dental Environmental Stress (DES) desenvolvido por Garbeeem 1980, sendo traduzido e validado para aplicação no Brasil, com as devidas adaptações culturais, por Sangiorgio (2013), sob o nome de Estresse no Ambiente Odontológico (EAO). Todos os itens foram avaliados segundo a escala Likert, variando de 0 (não aplicável/não pertinente), 1 (não estressante), 2 (ligeiramente estressante), 3 (moderadamente estressante) e 4 (muito estressante).

Após os dados serem coletados, eles foram tabulados, arquivados em planilhas eletrônicas (Excel $\circledast$ for Windows 2010) e posteriormente analisados no programa SPSS versão 20.0. Calculou-se a média da pontuação para cada estudante e depois obteve-se a média geral. Os dados foram submetidos a uma análise descritiva com verificação de frequências relativas e absolutas, médias e desvios padrões.

Os resultados do estudo foram obtidos através de análises com uma variável dependente relacionada às condições sociodemográficas e à situação acadêmica atual, sendo consideradas estatisticamente significantes quando o $\mathrm{p}$-valor foi $\leq 0,20$ na análise bivariada e $\leq 0,05$ no modelo de regressão logística. Para realizar as associações bivariadas e múltiplas, realizou-se a categorização da variável dependente em dois grupos: "abaixo ou igual à média" e "acima da média".

As limitações desta investigação consistiram prevalentemente ao método de estudo adotado, uma vez que, por ter se tratado de uma pesquisa com delineamento de corte transversal, de uso justificado pelo tempo reduzido, não houve a possibilidade de acompanhar os fatores estressores ao longo do tempo.

O estudo seguiu os cuidados éticos, sempre visando a proteção do indivíduo com base nas diretrizes e normas regulamentadas para a pesquisa envolvendo os seres humanos, estabelecidas pela Resolução no 466/2012 do Conselho Nacional de Saúde. O projeto foi aprovado no Comitê de Ética em Pesquisa da FUNORTE /SOEBRAS, com o parecer consubstanciado número 1.873.068. 


\section{RESULTADOS}

681 estudantes matriculados no curso de graduação em odontologia do primeiro ao décimo período, 345 foram convidados a participar voluntariamente do estudo e 344 aceitaram responder à pesquisa, perfazendo uma taxa de resposta de 99,7\%.

A caracterização dos acadêmicos considerou variáveis referentes às condições socioeconômicas, demográficas e a situação acadêmica atual. A maioria dos participantes foram do sexo feminino $(80,2 \%)$. A idade em anos dos educandos variou entre 18 a 53 anos com a média igual a 21,96 anos, mediana de 21 anos e com desvio padrão 3,92. A população de estudo apresentou-se em sua maioria com o estado civil solteiro $(93,3 \%)$, nos quais residem com os pais ou parentes em casa ou apartamento $(75,6 \%)$, tendo a necessidade de mudar de cidade (59,9\%), não possuem renda, uma vez que os gastos são financiados pelos pais ou outros $(91,3)$, não trabalham $(91,3 \%)$, dedicam ao estudo semanalmente excluindo-se horas de aula de 0 a 7 horas $(79,9 \%)$, permanecendo na mesma turma que ingressou no vestibular $(85,8 \%)$ e não tiveram reprovação nas disciplinas (77,9\%), conforme Tabela 1.

Diversos fatores estressores têm sido associados às características para o desenvolvimento da SB, e estes foram categorizados com as seguintes variáveis: Muito estressante (ME), Moderadamente estressante (BE), Ligeiramente Estressante (LE), Não Estressante (NE), Não pertinente/não aplicável (NP). De acordo com as respostas dos estudantes observou-se que o percentual de estresse obtido foram para ME $22 \%$, BE $19 \%$, LE $21 \%$, NE $23 \%$, e NA $15 \%$.

As variáveis que desencadeiam o fator mais estressante para o desenvolvimento do Burnout segundo as respostas dos participantes foram: provas e notas $(51,5 \%)$, falta de tempo para relaxar ou para lazer $(31,4 \%)$, completar os requisitos para graduação (notas/ produção clínica/ estágio) $(28,2 \%)$, conciliar vida pessoal com rotinas da faculdade $(32,8 \%)$, medo de reprovar em uma matéria ou perder o ano $(66,6 \%)$, insegurança em relação ao futuro profissional $(38,7 \%)$, responsabilidades financeiras $(48,3 \%)$, falta de tempo para fazer os trabalhos exigidos em sala de aula (32\%), medo de ser incapaz de recuperar em caso de ficar para trás nos estudos (36,9\%). As variáveis que se apresentaram como moderadamente estressante foram: quantidade de trabalho exigido em sala de aula $(43,3 \%)$ e falta de participação nos processos de tomada de decisão da faculdade $(27,3 \%)$.

Observou-se como variáveis ligeiramente estressante: falta de cooperação dos pacientes nos cuidados que devem tomar em casa $(27,3 \%)$, dificuldades em sala de aula $(31,7 \%)$, dificuldade de aprender procedimentos clínicos $(25,9 \%)$, receber críticas do trabalho realizado $(32,3 \%)$, dificuldades de aprender habilidades manuais de precisão exigidas no trabalho pré-clínico e de laboratório (28,5\%), diferenças entre as expectativas em relação a faculdade e realidade encontrada $(30,2 \%)$ e saúde física pessoal ( $29,4 \%$ ).

As variáveis julgadas como não estressantes foram: responsabilidades pelo cuidado com a saúde do paciente $(27 \%)$, competitividade por notas $(29,4 \%)$, ambiente criado pelo corpo docente $(31,4 \%)$, interações com o sexo oposto $(75,9 \%)$, falta de confiança em ser um estudante bem sucedido $(25,9 \%)$, falta de autoconfiança em ser um dentista bem sucedido (25,6\%), quantidade de desonestidades acadêmicas na faculdade $(22,7 \%)$, regras e regulamentos da faculdade de odontologia $(39,2 \%)$, atitudes discriminatórias da comunidade universitária em relação a estudantes do sexo feminino $(38,4 \%)$, conflito com a família sobre o futuro da profissão $(40,1 \%)$, e discriminação devido a raça, status social ou grupo étnico (34\%), conforme Tabela 2.

$\mathrm{Na}$ análise bivariada, considerando o nível de significância de $p<0,20$ para a condição ser analisada de forma múltipla, verificou-se que a presença dos fatores estressores foi determinada por algumas condições sociodemográficas e situações acadêmicas atuais, estando associadas significativamente ao fator estresse: "estar cursando a etapa clínica do curso de odontologia", "ter mudado de cidade para realizar o curso", "ter alguma reprovação nas disciplinas do curso" e "ter dedicado 8 ou mais horas de estudo semanalmente". Já na análise múltipla de regressão logística, as variáveis que alcançaram associação significativa com o nível de estresse foram: "etapa clínica no momento do curso", "teve reprovação em alguma disciplina do curso", "teve que mudar de cidade, estado ou pais para realização do curso" e "8 ou mais horas de estudo semanalmente", conforme apresentado na Tabela 3. 
Tabela 1 - Distribuição dos graduandos do curso de Odontologia quanto às características sociodemográficas e situação acadêmica atual. Montes Claros - MG, 2017. $(n=344)$

\begin{tabular}{|c|c|c|}
\hline CONDIÇÕES AVALIADAS & $\mathbf{N}$ & $\%$ \\
\hline \multicolumn{3}{|l|}{ Idade } \\
\hline Até 22 anos & 230 & 66,9 \\
\hline 23 anos ou mais & 114 & 33,1 \\
\hline \multicolumn{3}{|l|}{ Momento do curso } \\
\hline $1^{\circ}$ ano do Curso & 83 & 24,1 \\
\hline Etapa Pré-clínica & 52 & 15,1 \\
\hline Etapa Clínica & 172 & 50,0 \\
\hline Etapa Estágio & 37 & 10,8 \\
\hline \multicolumn{3}{|l|}{ Sexo } \\
\hline Feminino & 276 & 80,2 \\
\hline Masculino & 68 & 19,8 \\
\hline \multicolumn{3}{|l|}{ Estado Civil } \\
\hline Solteiro & 321 & 93,3 \\
\hline Casado & 21 & 6,1 \\
\hline Separado / Desquitado /Divorciado & 2 & 0,6 \\
\hline \multicolumn{3}{|l|}{ Condição de moradia atual } \\
\hline Mora sozinho & 84 & 24,4 \\
\hline Mora com outros & 260 & 75,6 \\
\hline \multicolumn{3}{|c|}{ Mudança de cidade, estado ou país para realização do curso } \\
\hline Não teve que mudar & 138 & 40,1 \\
\hline Teve que mudar & 206 & 59,9 \\
\hline \multicolumn{3}{|l|}{ Situação de renda } \\
\hline Não tem renda própria & 314 & 91,3 \\
\hline Tem renda própria & 30 & 8,7 \\
\hline \multicolumn{3}{|c|}{$\begin{array}{l}\text { Situação de trabalho (excluindo- se estágio, bolsa de pesquisa e } \\
\text { monitoria) }\end{array}$} \\
\hline Não trabalha & 314 & 91,3 \\
\hline Trabalha & 30 & 8,7 \\
\hline \multicolumn{3}{|c|}{ Horas de estudo dedicada semanalmente (excluindo- se horas de aula) } \\
\hline 0 a 7 horas estudo & 275 & 79,9 \\
\hline 8 ou mais horas estudo & 69 & 20,1 \\
\hline \multicolumn{3}{|c|}{ Permanece na mesma turma que ingressou no vestibular } \\
\hline Não & 49 & 14,2 \\
\hline Sim & 295 & 85,8 \\
\hline \multicolumn{3}{|c|}{ Já teve alguma reprovação nas disciplinas } \\
\hline Não & 268 & 77,9 \\
\hline Sim & 76 & 22,1 \\
\hline
\end{tabular}

Fonte: Veloso TPF, et al., 2020. 
Tabela 2 - Distribuição das respostas dos discentes quanto aos fatores estressores relacionados ao ambiente acadêmico. Montes Claros - MG, 2017. ( $\mathrm{n}=344$ ).

\begin{tabular}{|c|c|c|c|c|c|}
\hline FATOR ESTRESSOR & $\begin{array}{c}\text { Muito } \\
\text { estressante } \\
\mathbf{N}(\%) \\
\end{array}$ & $\begin{array}{c}\text { Moderadamente } \\
\text { estressante } \\
\mathbf{N}(\%) \\
\end{array}$ & $\begin{array}{c}\text { Ligeiramente } \\
\text { estressante } \\
\mathbf{N}(\%) \\
\end{array}$ & $\begin{array}{c}\text { Não estressante } \\
\mathrm{N}(\%) \\
\end{array}$ & $\begin{array}{c}\text { Não pertinente/ } \\
\text { aplicável } \\
\mathbf{N}(\%)\end{array}$ \\
\hline Quantidade de trabalho exigido em sala de aula & $60(17,4)$ & $149(43,3)$ & $95(27,6)$ & $40(11,6)$ & $0(0)$ \\
\hline Dificuldades em sala de aula & $47(13,7)$ & $93(27,0)$ & $109(31,7)$ & $84(24,4)$ & $11(3,2)$ \\
\hline Competitividade por notas & $88(25,6)$ & $58(16,6)$ & $52(15,1)$ & $101(29,4)$ & $45(13,1)$ \\
\hline Provas e notas & $17(51,5)$ & $88(25,6)$ & $50(14,5)$ & $23(6,7)$ & $6(1,7)$ \\
\hline Dificuldade de aprender procedimentos clínicos & $42(12,2)$ & $63(18,3)$ & $89(25,9)$ & $75(21,8)$ & $75(21,8)$ \\
\hline Ambiente criado pelo corpo docente & $47(13,7)$ & $64(18,6)$ & $102(29,7)$ & $108(31,4)$ & $23(6,7)$ \\
\hline Receber críticas do trabalho realizado & $55(16,0)$ & $59(17,2)$ & $111(32,3)$ & $101(29,4)$ & $18(5,2)$ \\
\hline $\begin{array}{l}\text { Dificuldade de aprender habilidades manuais de precisão exigidas no } \\
\text { trabalho pré-clínico e de laboratório }\end{array}$ & $42(12,2)$ & $57(16,6)$ & $98(28,5)$ & $91(26,5)$ & $56(16,3)$ \\
\hline Falta de confiança em ser um estudante de odontologia bem sucedido & $69(20,1)$ & $76(22,1)$ & $74(21,5)$ & $89(25,9)$ & $36(10,5)$ \\
\hline Falta de autoconfiança em ser um dentista bem sucedido & $69(20,1)$ & $73(21,2)$ & $73(21,2)$ & $88(25,6)$ & $41(11,9)$ \\
\hline Falta de tempo para relaxar ou para lazer & $108(31,4)$ & $80(23,3)$ & $73(21,2)$ & $64(18,6)$ & $19(5,5)$ \\
\hline Fraudes acadêmicas na faculdade (cola em provas, ou falsificações) & $75(21,8)$ & $71(20,6)$ & $71(20,5)$ & $78(22,7)$ & $49(14,2)$ \\
\hline Regras e regulamentos da faculdade de Odontologia & $34(9,9)$ & $63(18,3)$ & $100(29,1)$ & $135(39,2)$ & $12(3,5)$ \\
\hline $\begin{array}{l}\text { Completar os requisitos para graduação (notas, produção clínica e } \\
\text { estágio) }\end{array}$ & $97(28,2)$ & $76(22,1)$ & $78(22,7)$ & $53(15,4)$ & $40(11,6)$ \\
\hline Conciliar vida pessoal com as rotinas da faculdade & $113(32,8)$ & $87(25,3)$ & $94(27,3)$ & $47(13,7)$ & $3(0,9)$ \\
\hline $\begin{array}{l}\text { Diferenças entre as expectativas em relação à faculdade e a realidade } \\
\text { encontrada }\end{array}$ & $78(22,7)$ & $94(27,3)$ & $104(30,2)$ & $55(16,0)$ & $13(3,8)$ \\
\hline $\begin{array}{l}\text { Falta de participação nos processos de tomada de decisão da } \\
\text { faculdade }\end{array}$ & $63(18,3)$ & $94(27,3)$ & $86(25,0)$ & $71(20,6)$ & $30(8,7)$ \\
\hline Medo de reprovar em uma matéria ou perder o ano & $229(66,6)$ & $48(14)$ & $36(10,5)$ & $22(6,4)$ & $9(2,6)$ \\
\hline Insegurança em relação ao futuro profissional & $133(38,7)$ & $83(24,1)$ & $70(20,3)$ & $45(13,1)$ & $13(3,8)$ \\
\hline Responsabilidades financeiras & $166(48,3)$ & $70(20,3)$ & $57(16,6)$ & $30(8,7)$ & $31(6,1)$ \\
\hline Falta de tempo para fazer os trabalhos exigidos em sala de aula & $110(32,0)$ & $81(23,5)$ & $93(27,0)$ & $43(12,5)$ & $17(4,9)$ \\
\hline Saúde física pessoal & $76(22,1)$ & $53(15,4)$ & $101(29,4)$ & $100(29,1)$ & $14(4,1)$ \\
\hline Discriminação devido à raça, status social ou grupo étnico & $57(16,6)$ & $18(5,2)$ & $40(11,6)$ & $117(34,0)$ & $112(32,6)$ \\
\hline Medo de ser incapaz de se recuperar ao ficar para trás nos estudos & $127(36,9)$ & $65(18,9)$ & $62(18,0)$ & $47(13,7)$ & $43(12,5)$ \\
\hline
\end{tabular}

Fonte: Veloso TPF, et al., 2020. 


\section{Revista Eletrônica Acervo Saúde / Electronic Journal Collection Health | ISSN 2178-2091}

Tabela 3: Resultado das análises bivariada e múltipla relativas aos fatores associados ao estresse no ambiente acadêmico do curso de Odontologia da FUNORTE. Montes Claros- Minas Gerais, $2017(\mathrm{n}=344)$.

\begin{tabular}{|c|c|c|c|c|c|}
\hline \multirow[b]{2}{*}{ Condições } & \multicolumn{3}{|c|}{ Análise bivariada } & \multicolumn{2}{|c|}{ Análise múltipla } \\
\hline & $\begin{array}{c}\text { Abaixo ou igual à } \\
\text { média } \\
\mathbf{n}(\%)\end{array}$ & $\begin{array}{c}\text { Acima da média } \\
\mathrm{n}(\%) \\
\end{array}$ & $p$-valor & $\begin{array}{l}\text { Modelo } \\
\text { ajustado } \\
\text { (IC95\%) }\end{array}$ & $p$-valor \\
\hline \multicolumn{6}{|l|}{ Idade } \\
\hline Até 22 anos & $110(47,8)$ & $120(52,2)$ & \multirow{2}{*}{0,401} & - & - \\
\hline 23 anos ou mais & $60(52,6)$ & $54(47,4)$ & & - & - \\
\hline \multicolumn{6}{|l|}{ Momento do curso } \\
\hline $1^{\circ}$ ano do Curso & $50(60,2)$ & $33(39,8)$ & \multirow{4}{*}{0,002} & 1 & \\
\hline Etapa Pré-clínica & $34(65,4)$ & $18(34,6)$ & & $0,74(0,35 ; 1,56)$ & 0,437 \\
\hline Etapa Clínica & $69(40,1)$ & $103(59,9)$ & & $1,96(1,12 ; 3,43)$ & 0,019 \\
\hline Etapa Estágio & $17(45,9)$ & $20(54,1)$ & & $1,37(0,61 ; 3,08)$ & 0,442 \\
\hline \multicolumn{6}{|l|}{ Sexo } \\
\hline Feminino & $134(48,6)$ & $142(51,4)$ & \multirow{2}{*}{0,517} & - & - \\
\hline Masculino & $36(52,9)$ & $32(47,1)$ & & - & - \\
\hline \multicolumn{6}{|l|}{ Estado Civil } \\
\hline Solteiro & $158(49,2)$ & $163(50,8)$ & \multirow{3}{*}{0,961} & - & - \\
\hline Casado & $11(52,4)$ & $10(47,6)$ & & - & - \\
\hline Separado/desquitado/divorciado & $1(50,0)$ & $1(50,0)$ & & - & - \\
\hline \multicolumn{6}{|l|}{ Condição de moradia atual } \\
\hline Mora sozinho & $42(50,0)$ & $42(50,0)$ & \multirow[b]{2}{*}{0,902} & - & - \\
\hline Mora com outros & $128(49,2)$ & $132(50,8)$ & & - & - \\
\hline \multicolumn{6}{|l|}{ Mudança de cidade, estado ou pais para realização do curso } \\
\hline Não teve que mudar & $79(57,2)$ & $59(42,8)$ & \multirow{2}{*}{0,017} & 1 & \\
\hline Teve que mudar & $91(44,2)$ & $115(55,8)$ & & $1,73(0,61 ; 3,08)$ & 0,018 \\
\hline \multicolumn{6}{|l|}{ Situação de renda } \\
\hline Não tem renda própria & $155(49,4)$ & $159(50,6)$ & \multirow{2}{*}{0,947} & - & - \\
\hline Tem renda própria & $15(50,0)$ & $15(50,0)$ & & - & - \\
\hline \multicolumn{6}{|c|}{ Situação de trabalho (excluindo- se estágio, bolsa de pesquisa e monitoria) } \\
\hline Não trabalha & $154(49,0)$ & $160(51,0)$ & \multirow{2}{*}{0,654} & - & - \\
\hline Trabalha & $16(53,3)$ & $14(46,7)$ & & - & - \\
\hline \multicolumn{6}{|c|}{ Horas de estudo dedicada semanalmente (excluindo- se horas de aula) } \\
\hline 0 a 7 horas estudo & $128(46,5)$ & $147(53,5)$ & \multirow{2}{*}{0,033} & 1 & \\
\hline 8 ou mais horas estudo & $42(60,9)$ & $27(39,1)$ & & $0,55(0,31 ; 0,96)$ & 0,038 \\
\hline \multicolumn{6}{|l|}{ Permanece na mesma turma que ingressou no vestibular } \\
\hline Não & $23(46,9)$ & $26(53,1)$ & \multirow{2}{*}{0,708} & - & - \\
\hline Sim & $147(49,8)$ & $148(50,2)$ & & - & - \\
\hline \multicolumn{6}{|l|}{ Já teve alguma reprovação nas disciplinas } \\
\hline Não & $142(53,0)$ & $126(47,0)$ & \multirow{2}{*}{0,013} & 1 & \\
\hline Sim & $28(36,8)$ & 48 & & $1,66(0,95 ; 2,90)$ & 0,076 \\
\hline
\end{tabular}

Fonte: Veloso TPF, et al., 2020 


\section{DISCUSSÃO}

Esta investigação permitiu o conhecimento das condições no curso de Odontologia que submetem os acadêmicos ao estresse que estão associados a SB quando estes permanecem rotineiramente. $O$ estudo foi o primeiro a abordar os fatores estressores entre os estudantes do curso de graduação em odontologia. Verificou-se, que é um tema que há necessidade de maior exploração cientifica. Tal estudo foi executado de forma precisa com uma amostra específica, ou seja, foi realizado entre os educandos devidamente matriculados na Instituição de Ensino. O Curso de Odontologia da FUNORTE é dividido em 10 semestres. Cada semestre aborda diferentes conhecimentos e habilidades acadêmicas, preparando os estudantes para um futuro clínico profissional melhor.

Diante dos resultados analisados comparando se as buscas em artigos e periódicos se certifica que há relação das condições sociodemográficas e situação acadêmica atual com os dados obtidos na literatura. Num estudo realizado em Santa Catarina com 282 estudantes, houve uma predominância do sexo feminino, com estado civil solteiro, residindo com os pais ou outros, tendo a necessidade de mudar de cidade, permanecendo na mesma turma que ingressou no vestibular, não possuem renda, não trabalham no momento, exceto as horas de estudo nos quais os universitários catarinense dedicam-se a mais nos estudos semanalmente excluindo-se as horas de aula (SPIGER V, 2015). Tais resultados também coincidiram com outros estudos em outras áreas da saúde como o da pesquisa realizada em Santa Catarina com acadêmicos de enfermagem, nos quais a população preponderou-se no sexo feminino, com estado civil solteiro e que também residiam com suas famílias (TOMASCHEWSKI-BARLEM JG, et al., 2014).

No que diz respeito ao estresse, observa-se que, a prevalência deste ou a complexidade em encará-lo têm sido indicada como fator colaborador para o aparecimento da Síndrome de Burnout, condição que está relacionada apenas ao ambiente laboral, caracterizando em um estágio mais evoluído da exposição ao estresse psicológico crônico (CAMPOS JADB, et al., 2012). Expor-se ao estresse no ambiente acadêmico parece ser um importante preditor do estresse crônico (POZOS-RADILLO BE, et al., 2014).

Em relação aos fatores estressores, os estudantes de Odontologia da FUNORTE apresentaram-se com algum grau de estresse nas variáveis utilizadas. Após calcular a média para cada estudante segundo o instrumento utilizado, encontrou-se a média geral de 2,11 , com desvio padrão 0,65 . Verificou-se que os mesmos se encontram entre os níveis não estressante e muito estressante conforme as exigências que 0 próprio ensino odontológico oferece dentre outros fatores associados. A média geral desses fatores encontrou-se inferior em relação a outros estudos desenvolvidos como o da Turquia onde obteve-se um média de 2,5 (URAZ A, et al., 2013).

Num estudo realizado em Londrina com acadêmicos de Odontologia, observou-se que os principais fatores estressores que atingem os mesmos são: atividades avaliativas, provas semestrais, o que gera maior tensão, falta de recursos financeiros para obter a lista de matérias exigidas pela instituição, contratempo para se relacionar com familiares e amigos, descrença em realizar suas tarefas práticas e teóricas, falta de entusiasmo, falta de reforço positivo e incapacidade de se relacionar com os colegas de turma e educadores (ROVIDA TAS, 2015).

De acordo com um estudo realizado em uma faculdade no estado de São Paulo, os períodos iniciais e finais da graduação são estaticamente significantes a presença de cansaço emocional, desnorteamento e ineficácia profissional. Quando se compara os graduandos dos primeiros períodos com os dos períodos finais, a prevalência das manifestações da SB é maior nos estudantes dos períodos finais, uma vez que estes enfrentam uma maior carga horária de atividades, como o Trabalho de Conclusão de Curso e estágios supervisionados para obtenção da titulação desejada (FOGAÇA MC, et al., 2012). Diante disso, conforme o avanço gradativo dos períodos subsequentes, as exigências que o curso de Odontologia prepondera aumentam conforme as etapas do primeiro período, pré-clínica (segundo ao terceiro período), clínicas (quinto ao nono período) e décimo período (estágio) ao longo dos 10 semestres letivos.

$\mathrm{Na}$ etapa inicial, ou seja, no primeiro ano da graduação, os fatores encontrados capazes de desenvolver o estresse são: dificuldade de adaptação no período de transição do ensino médio para o ensino superior, preocupação quanto à demanda das atividades acadêmicas iniciais, além da relação entre colegas e 
professores (BRITO BJQ, et al., 2014; LANGOSKI JE, et al., 2014). Nessa etapa o percentual de estresse acima da média foi de $39,8 \%$ e esse valor ocupou a terceira posição no que se refere a associação ao grau de estresse na academia investigada.

Considerando-se os estudantes da etapa pré-clínica, o percentual de estresse acima da média foi identificado em $34,6 \%$ do grupo. Diante dos resultados obtidos, certificou-se que essa etapa foi a que se apresentou menor grau de associação ao estresse capaz de desenvolver a SB. Esse resultado diverge de outros estudos na literatura, como a pesquisa realizada em Santa Catarina, onde a etapa pré-clínica manifestou-se com uma taxa de estresse superior, o que pode se justificar pela complexidade do estudante aprender os procedimentos laboratoriais e executá-los nessa etapa da formação (SPIGER V, 2015).Sugerese que os possíveis motivos para tal divergência com outros estudos possam estar relacionados à organização das práticas pré-clínicas, estrutura física, ambiente criado pelo corpo docente e suporte que a instituição de ensino oferece.

$\mathrm{Na}$ análise da etapa clínica, observou-se que o percentual de estresse foi superior à média em relação as outras etapas, com um valor de 59,9\%. Esse percentual está relacionado com outros resultados na literatura, sendo associado à maior carga horaria de atividades teóricas para o Trabalho de Conclusão de Curso, atividades clínicas ou práticas, dificuldade de conciliar vida acadêmica com a social e/ou conjugal, provas e notas o que gera maior tensão, medo de se reprovar em uma matéria ou perder o ano, insegurança com relação ao futuro profissional, responsabilidades financeiras, falta de tempo para fazer os trabalhos exigidos em sala de aula, medo de ser incapaz em se recuperar em caso de ficar para trás nos estudos (SANGIORGIO JPM, 2013).

Logo no período de estágio, ou seja, na etapa final do curso (décimo período), obteve-se um percentual de $54,1 \%$, ocupando a segunda posição no percentual acima da média dos fatores estressores. As variáveis capazes de desencadear esse valor podem estar relacionadas a preocupação em completar os requisitos para a graduação, falta de tempo para relaxar ou para lazer, insegurança quanto ao futuro profissional, responsabilidades financeiras, falta ou atraso dos pacientes nas consultas, grande carga horaria clínica, além de ser um período que está voltado para as responsabilidades de um profissional (URAZ A, et al., 2013).

Os resultados dessa investigação apresentam validade interna, pois houve uma elevada taxa de resposta, porém, podem ser superestimados, levando em conta o relato dos acadêmicos, que podem optar por respostas consideradas certas, como as horas de estudo dedicadas semanalmente, mesmo que elas não representem a realidade de sua prática. Logo, para minimizar esse viés, as entrevistadoras esclareceram aos participantes sobre a confidencialidade das informações coletadas.

\section{CONCLUSÃO}

O estudo permitiu identificar os principais fatores estressores associados à SB entre os estudantes do curso de Odontologia. Os achados podem estar relacionados ao fato desses alunos enfrentam diariamente diversas condições que elevam o nível de estresse desde o primeiro período até a etapa clínica, quando eles se deparam com o maior grau de estresse. Dessa forma, as condições que expõe os acadêmicos ao estresse podem levar ao desenvolvimento das manifestações dos sinais e sintomas clínicos da Síndrome de Burnout, o que pode ser prevenido e acompanhado através do amplo conhecimento do tema e a elaboração de ações institucionais direcionadas a melhorar a qualidade de vida dos estudantes.

\section{REFERÊNCIAS}

1. BALOGUN J, et al. Test-retes treability of a psychometric instrument designed tomeasure physicaltherapy student's Burnout. Perceptual and Motor Skill, Missoula, 1995; 81: 667-672.

2. BORINE RCC, et al. Relação entre a qualidade de vida e o estresse em acadêmicos da área da saúde. Estudos Interdisciplinares em Psicologia, Londrina, 2015; 6(1): 100-118.

3. BRASIL. Conselho Nacional de Saúde. Resolução $n^{\circ} 466$, de 12 de dezembro de 2012. Aprova normas regulamentadoras de pesquisas envolvendo seres humanos. Brasília: Diário Oficial da União, 2013.

4. BRITO BJQ, GORDIA AP, QUADROS TMB. Literature review on the life style of colleg estudents. Revista Bras Qual Vida, 2014; 6(2): 66-76. 
5. CAMPOS JADB, et al. Síndrome de Burnout em graduandos de Odontologia. Revista Brasileira de Epidemiologia, São Paulo, 2012; 15(1): 155-165.

6. CAMPOS JADB, MAROCO J. Adaptação transcultural Portugal-Brasil do Inventário de Burnout de Maslach para estudantes. Rev. de Saúde Pública, São Paulo, 2012; 46(5): 816-824.

7. DIEHL L, CARLOTTO MS. Síndrome de Burnout: indicadores para a construção de um diagnóstico. Psicologia Clinica, Rio de Janeiro, 2015; 27(2): 161-179.

8. FOGAÇA MC, et al. Burnout em estudantes de psicologia: diferenças entre alunos iniciantes e concluintes. Revista Aletheia, 2012; 38-39:124-131.

9. GARBEE JR WH. Sources of stress in the dental school environment. LDA J, Winter, 1981; 39(4): 9-14.

10. GARBEE JR WH, ZUCKER SB, SELBY GR. Perceivedsourcesof stress among dental students. J Am Dent Assoc, 1980; 100(6): 853-7.

11. GOMES RC. Síndrome de Burnout em docentes. Trabalho de Conclusão de Curso (Graduação em Odontologia). Universidade Estadual da Paraíba, Campina Grande, 2014; 30 p.

12. KOGA GKC, et al. Fatores associados a piores níveis na escala de Burnout em professores da educação básica. Cad. Saúde Coletiva, Rio de Janeiro, 2015; 23(3): 268-275.

13. LANGOSKI JE, et al. Influência da trajetória acadêmica sobre o estresse e a percepção de estudantes de Odontologia. Revista de Odontologia da UNESP, 2014, 43: 253.

14. MASLACH C, LEITER MP. The truth about Burnout. Howorganizations cause personal stress and whatto do about it. San Francisco: Jossey-BassInc; 1997.

15. NEVES CP. Burnout em estudantes de graduação em odontologia. TCC (Graduação) - Curso de Odontologia, Centro De Ciências da Saúde. Universidade Federal de Santa Catarina, Florianópolis, 2014; 89 p.

16. PEREIRA AMTB. Burnout: quando o trabalho ameaça o bem-estar do trabalhador. 4ª ed. São Paulo: Casa do Psicólogo, 2010.

17. POZOS-RADILLO BE, et al. Academic stress as a predictorofchronic stress in universitystudents. Psicología educativa, $2014 ; 20(1): 47-52$.

18. ROVIDA TAS. Estresse e o estilo de vida dos acadêmicos ingressantes em um curso de graduação em Odontologia. Rev. ABENO, Londrina, 2015; 15(3): 26-34.

19. SANGIORGIO JPM. Estresse em odontologia: Adaptação transcultural e validação do Dental Environmental Stress para estudantes de odontologia brasileiros. Dissertação (Mestrado) - Curso de Odontologia. Universidade Estadual de Londrina, Londrina, 2013; 62 p.

20. SCHMIDT DRC, et al. Qualidade de vida no trabalho e Burnout em trabalhadores de enfermagem de Unidade de Terapia Intensiva.Revista Brasileira de Enfermagem, Brasília, 2013; 66(1): 13-7.

21. SPIGER V. Fatores estressores entre estudantes do Curso de Graduação em Odontologia da UFSC. TCC (Graduação) - Curso de Odontologia, Departamento de Odontologia. Universidade Federal de Santa Catarina, Florianópolis, 2015; 82 p.

22. TAVARES KFA, et al. Ocorrência da síndrome de Burnout em enfermeiros residentes. Revista Acta paulista de Enfermagem, São Paulo, 2014; 27(3): 260-265.

23. TOMASCHEWSKI-BARLEM JG, et al. Opção e evasão de um curso de graduação em enfermagem: percepção de estudantes evadidos. Revista Gaúcha de Enfermagem, Porto Alegre, 2012; 3(2): 132-138.

24. TOMASCHEWSKI-BARLEM JG, et al. Síndrome de Burnout entre estudantes de graduação em enfermagem de uma universidade pública. Revista Latino-Am. Enfermagem, Rio Grande do Sul, 2014; 22(3): 754-762.

25. URAZ A, et al. Psychologicalwell-being, health, and stress sources in Turkish dental students. J Dent Educ, 2013; 77(10): 1345-1355.

26. VARELLA, D. Doenças e sintomas: síndrome de Burnout. set. 2016. Disponível em: <http://drauziovarella.com.br/letras/s/sindrome-de-burnout/>. Acesso em: 20 dez. 2019.

27. VIANA GM, et al. Relação entre Síndrome de Burnout, ansiedade e qualidade de vida entre estudantes de ciências da saúde. Revista da Universidade Vale do Rio Verde, Três Corações, 2014; 12(1): 876-885. 